\title{
Publisher Correction: Stem cells: The reprogramming method matters
}

Thomas F. Allison and William E. Lowry

Correction to: Nature Biomedical Engineering https://doi.org/10.1038/s41551-017-0148-z (2017); published online 10 October 2017.

In the version of this News and Views article originally published, an erroneous hyphen was included in the term thuman-induced pluripotent stem cells'; it should have read 'human induced pluripotent stem cells'. This error has now been corrected in all versions of the News and Views article.

Published online: 2 January 2018

https://doi.org/10.1038/s41551-017-0170-1 